Check for updates

Cite this: Mater. Chem. Front. 2020, 4, 3660

Received 21st June 2020, Accepted 28th September 2020

DOI: $10.1039 / \mathrm{d} 0 \mathrm{qm} 00418 \mathrm{a}$

rsc.li/frontiers-materials

\section{Pendant conjugated molecules based on a heterogeneous core structure with enhanced morphological and emissive properties for organic semiconductor lasing $\dagger$}

\author{
Xu Liu, $\ddagger^{a}$ Ming Sang, $\ddagger^{a}$ Jinghan Zhou, ${ }^{a}$ Shihao Xu, ${ }^{a}$ Jialing Zhang, ${ }^{a}$ Yu Yan, ${ }^{a}$ He Lin ${ }^{a}$ \\ and Wen-Yong Lai (D) *ab
}

\begin{abstract}
A family of oligofluorene-type pendant conjugated molecules ( $\mathbf{C}$ 1 and $\mathbf{C 2}$ ), consisting of a triazine center with two diphenylamine and one carbazole heterogeneous substituents to form a donor-acceptor (D-A) core structure, are synthesized and characterized. The relationship between the molecular architectures and the corresponding functional properties was systematically investigated by varying the fluorene lengths and the substituents on the triazine center and by comparing with the linear oligomer counterparts (xFCz), the pendant model compound (DNPhCzT) and the analogue with a homogeneous pendant core structure $(T-m)$. The resulting pendant conjugated molecules exhibited a depressed crystallization tendency and thus enhanced morphology stability due to the incorporation of a bulky heterogeneous pendant core structure. Remarkably, enhanced photoluminescence quantum yields (PLQYs) (0.76 for C1 and 0.61 for C2) were afforded by virtue of the integration of bulky diphenylamine-carbazole heterogeneous core structures, in comparison with their homogeneous pendant analogue T-m (0.58). Solution-processed neat films demonstrated promising amplified spontaneous emission (ASE). The lowest ASE threshold ( $E_{\mathrm{th}}^{\mathrm{ASE}}$ ) for the non-doped films was recorded as $5.0 \mu \mathrm{J} \mathrm{cm}^{-2}$ and $6.4 \mu \mathrm{J} \mathrm{cm}^{-2}$ for $\mathbf{C} \mathbf{1}$ and $\mathbf{C 2}$, respectively. The $E_{\mathrm{th}}^{\mathrm{ASE}}$ was almost unchanged for $\mathrm{C} 1$ upon annealing from room temperature to $150{ }^{\circ} \mathrm{C}$, while the $E_{\mathrm{th}}^{\mathrm{ASE}}$ of the linear counterparts exhibited a sudden increase above $100{ }^{\circ} \mathrm{C}$. Compared to $\mathbf{C 2}$ with extended oligofluorene arms, C1 with one fluorene arm exhibited a lower $E_{\mathrm{th}}^{\mathrm{ASE}}$ and better optical stability at high temperature. The results suggest that excellent optoelectronic properties and enhanced thermal and morphological stabilities are afforded by constructing pendant conjugated molecules with a bulky heterogeneous core structure, and these materials have great potential as gain media for organic lasing.
\end{abstract}

\section{Introduction}

Great development made in the molecular design and synthesis of organic semiconductors has contributed to the advancement of optoelectronic devices, such as organic light-emitting diodes (OLEDs) ${ }^{1,2}$ organic field-effect transistors (OFETs), ${ }^{3,4}$ organic

\footnotetext{
${ }^{a}$ Key Laboratory for Organic Electronics and Information Displays (KLOEID), Institute of Advanced Materials (IAM), Nanjing University of Posts \& Telecommunications (NUPT), 9 Wenyuan Road, Nanjing, 210023, China. E-mail:iamwylai@njupt.edu.cn

${ }^{b}$ Frontiers Science Center for Flexible Electronics (FSCFE), MIIT Key Laboratory of Flexible Electronics (KLoFE), Shaanxi Key Laboratory of Flexible Electronics, Xi'an Key Laboratory of Flexible Electronics, Xi'an Key Laboratory of Biomedical Materials \& Engineering, Xi'an Institute of Flexible Electronics, Institute of Flexible Electronics (IFE), Northwestern Polytechnical University, Xi'an 710072, Shaanxi, China

$\dagger$ Electronic supplementary information (ESI) available. See DOI: 10.1039/ doqm00418a

\$ These authors contributed equally to this work.
}

photovoltaics (OPVs), ${ }^{5}$ and organic semiconductor lasers (OSLs). ${ }^{6,7}$ Among them, advances in OSLs based on organic semiconductors are particularly challenging. ${ }^{8}$ Although extensive efforts have been concentrated on optically pumped organic lasing, electrically pumped OSLs, which require high carrier mobilities, a relatively high current density and superior stability, still face great challenges. ${ }^{9}$ With the aim to realize electrically pumped OSLs, great attention has been paid to the exploration of novel organic gain media with excellent carrier mobilities, superior optical gains and high thermal stability. ${ }^{10-16}$ However, developing efficient gain media simultaneously with improved electrical properties, high luminous efficiency and low lasing thresholds is quite challenging. It is critical to elucidate the structurefunction relationships with the aim to establish a rational molecular design strategy for exploring robust organic gain media for OSLs.

Achieving electrically pumped organic lasing requires not only excellent optical gain properties but also good electrical 
properties for organic gain media. To the best of our knowledge, the optimization of current carrier injection and transport properties is favorable to balance the carrier injection and transport. For this purpose, electron-donating groups and/or electron-accepting moieties have been incorporated into the molecular structures to modulate the highest occupied molecular orbital (HOMO) and/or the lowest unoccupied molecular orbital (LUMO), thus enhancing the hole and/or electron injection and transport, respectively. ${ }^{17}$ Recently, we have exploited a family of starbursts comprising a truxene core and oligofluorene bridges by introducing diphenylamine (DPA) units as end-cappers, affording enhanced hole injection and transport and robust lasing properties. ${ }^{18}$ It is promising to improve the electron and hole injection and transport simultaneously by incorporating electronaccepting and electron-donating moieties into one molecular structure. ${ }^{19}$ Unfortunately, the construction of donor-acceptor (D-A) molecules generally manifests undesirable intramolecular charge transfer (ICT) characteristics, which result in drawn-out estimated fluorescence lifetimes and inferior luminous efficiency due to the inhibitory electronic transitions between the spatially separated orbitals. ${ }^{20-23}$ Nevertheless, from a fundamental viewpoint, high photoluminescence quantum yields (PLQYs) and short exciton lifetimes are believed to be helpful to achieve stimulated emission with low amplified spontaneous emission (ASE) thresholds. ${ }^{24,25}$ Consequently, it is generally highly desirable to explore novel organic gain media that not only have excellent optical properties for stimulated emission but also possess enhanced electrical properties for carrier injection and transport.

Here, we present the synthesis and optical gain properties of a set of oligofluorene-type pendant conjugated molecules (C1 and C2) with simultaneously incorporated electrondonating and electron-accepting moieties but with depressed ICT properties to afford improved electrical properties while maintaining excellent optical gain properties. 1,3,5-Triazine was chosen as the core due to its high electron-transport property, electron-accepting characteristics and enhanced thermal stability. ${ }^{26}$ Two diphenylamine and one carbazole were introduced into the triazine core to afford a heterogeneous D-A structure to finely adjust the electrical properties, depress intermolecular interactions and enhance the morphological properties. $^{27,28}$ The relationship between the molecular structures and the corresponding functional properties was systematically investigated by varying the fluorene lengths and the substituents on the triazine center. Their thermostability, optical, electrochemical, morphology and ASE characteristics were studied in detail in comparison with those of the pendant model compound (DNPhCzT), linear oligomers (xFCz) and the analogue with a homogeneous pendant core structure (T-m). Consequently, improved thermal and optical stability, excellent film morphology, higher PLQYs, lower ASE thresholds and enhanced ASE stability were achieved. The results suggest that constructing pendant conjugated molecules with a bulky heterogeneous core structure is beneficial for accomplishing not only enhanced film morphology but also good electrical and optical properties, demonstrating the great potential of these materials as robust gain media for OSLs.

\section{Results and discussion}

\section{Synthesis and characterization}

The synthetic routes of the oligofluorene-type pendant conjugated molecules are shown in Scheme 1. We completed the synthesis of a triazine-centered derivative 2 by successive twostep nucleophilic substitution. According to previous reported processes, the target compounds $\mathbf{C} \mathbf{1}$ and $\mathbf{C 2}$ were obtained by Suzuki cross-coupling reactions. Their chemical structures and purities were confirmed using ${ }^{1} \mathrm{H}$ and ${ }^{13} \mathrm{C}$ NMR measurement and MALDI-TOF mass spectrometry (Fig. S1-S6, ESI $\dagger$ ). C1 and C2 exhibited good solubility in dichloromethane, toluene, tetrahydronfuran, chlorobenzene and chloroform.

\section{Thermal and morphology properties}

With the aim to investigate the thermal stabilities of the resulting pendant conjugated molecules (Cx), thermogravimetric analysis (TGA) and differential scanning calorimetry (DSC) data were recorded in a $\mathrm{N}_{2}$ atmosphere. C1 and C2 demonstrated high thermostability with their decomposition temperature $\left(T_{\mathrm{d}}\right)$ with $5 \%$ weight loss of up to $426{ }^{\circ} \mathrm{C}$ and $405{ }^{\circ} \mathrm{C}$, respectively (Fig. 1a). As a comparison, $T_{\mathrm{d}}$ of the linear oligomers $2 \mathbf{F C z}$ and $4 \mathbf{F C z}$ was recorded as $393{ }^{\circ} \mathrm{C}$ and $374{ }^{\circ} \mathrm{C}$, respectively. The pendant conjugated molecules $\mathbf{C x}$ exhibited no distinct melting/crystallization process or glass phase transition during the repeated scanning cycle (Fig. 1b). In comparison, the linear oligomers $\mathbf{2 F C z}$ and $\mathbf{4 F C z}$ exhibited a glassy phase transition with the glass transition temperature $\left(T_{\mathrm{g}}\right)$ of $64{ }^{\circ} \mathrm{C}$ and $75{ }^{\circ} \mathrm{C}$, respectively (Fig. 1b). Wide-angle X-ray diffraction (WAXD) patterns of the powders (Fig. S10, ESI $\dagger$ ) showed obviously depressed crystalline properties for the pendant conjugated molecules $\mathbf{C x}$, in sharp contrast to those of the linear oligomers $\mathbf{x F C z}$, which exhibited distinct crystalline signals. The results suggest that the strategy of designing pendant conjugated molecules with a bulky heterogeneous core structure is beneficial to improving thermal stabilities and depressing crystallinity.

Atomic force microscopy (AFM) measurements of C1 and C2 were recorded to explore the surface morphology (Fig. 2). Spincoated thin films of Cx showed a smooth surface morphology with a root-mean-square (RMS) roughness of $0.2 \mathrm{~nm}$ for both $\mathbf{C 1}$ and $\mathbf{C 2}$. While the films of $\mathbf{2 F C z}$ and $\mathbf{4 F C z}$ exhibited a similar surface with a higher RMS roughness $(0.3 \mathrm{~nm})$. Compared with the analogue T-m based on a homogeneous core structure, ${ }^{29}$ the resulting pendant molecules $\mathbf{C 1}$ and $\mathbf{C 2}$ based on the heterogeneous core structures exhibited enhanced surface morphology with a smaller RMS, which may originate from the depressed intermolecular stacking induced by the incorporation of DPA substituents (Fig. S7-S9, ESI $\dagger$ ). The observation implies that the pendant conjugated molecules with heterogeneous core structures show enhanced amorphous film properties.

\section{Photophysical properties}

Fig. 3 depicts the normalized UV-Vis absorption and photoluminescence (PL) spectra of $\mathbf{C x}$ and $\mathbf{x F C z}$ in solutions and spin-coated thin films (Table 1). In THF solutions, C1 and C2 

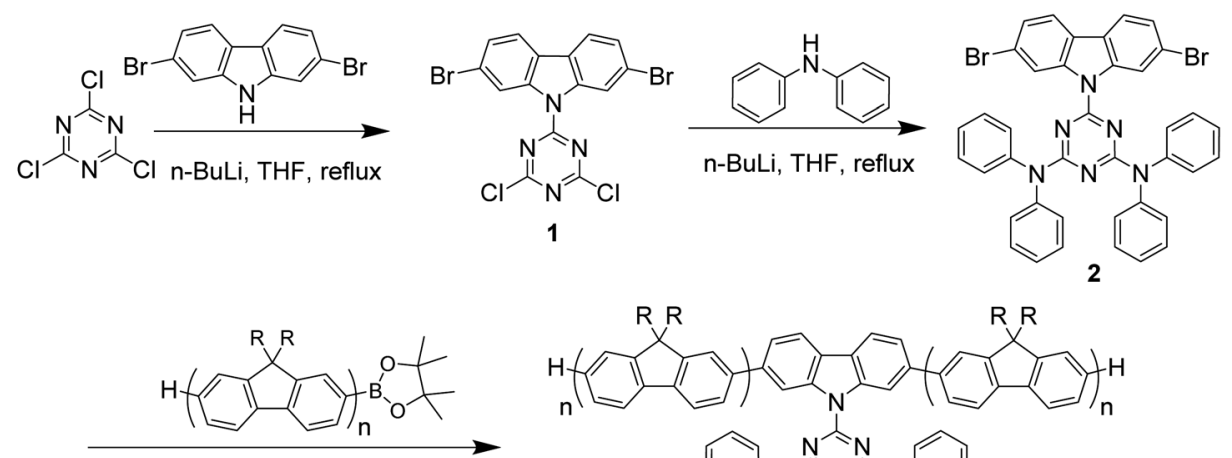

toluene, $2 \mathrm{M} \mathrm{K}_{2} \mathrm{CO}_{3}, \mathrm{H}_{2} \mathrm{O}$, $\mathrm{Pd}\left(\mathrm{PPh}_{3}\right)_{4}, 100^{\circ} \mathrm{C}$.
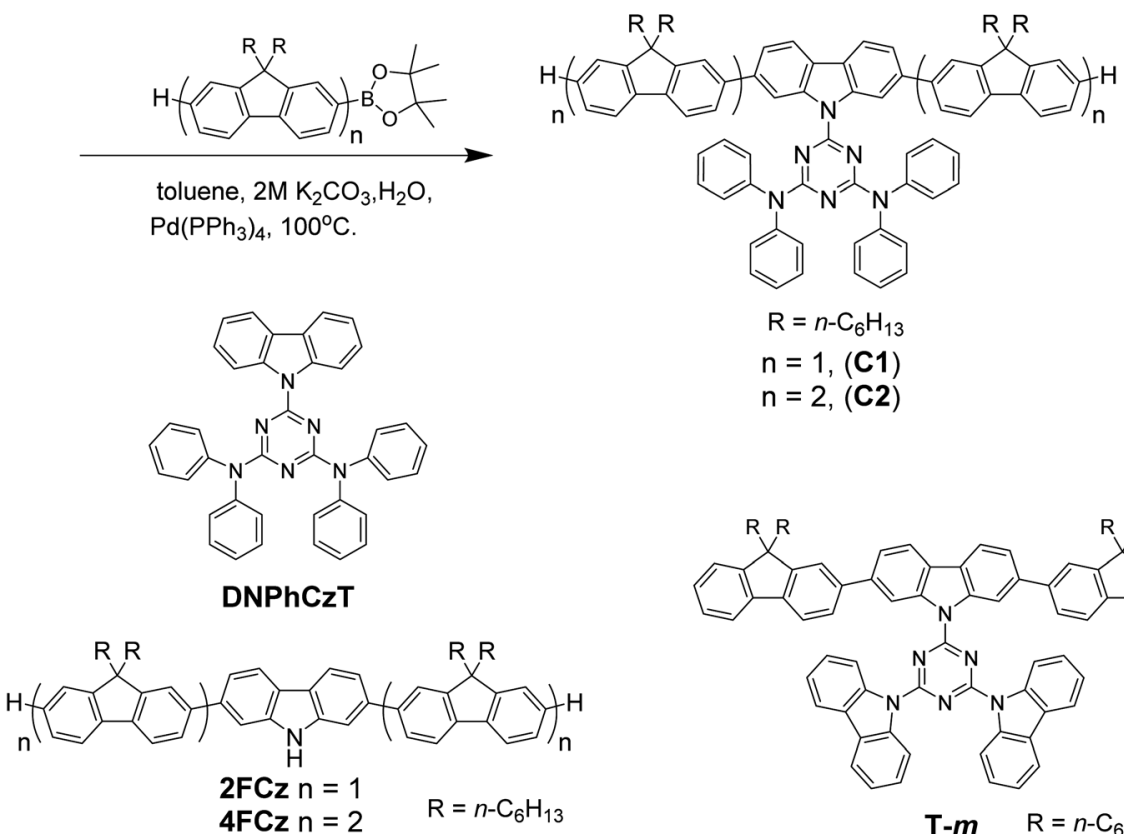

$$
\begin{aligned}
\mathrm{R} & =n-\mathrm{C}_{6} \mathrm{H}_{13} \\
\mathrm{n} & =1,(\mathbf{C} 1) \\
\mathrm{n} & =2,(\mathbf{C} 2)
\end{aligned}
$$

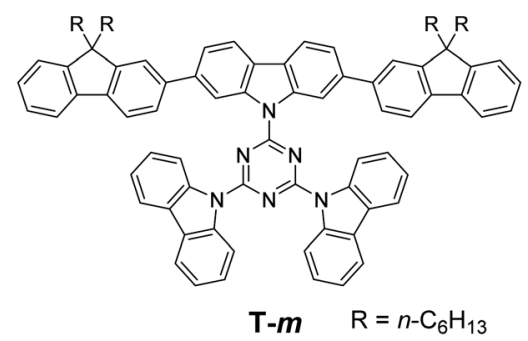

Scheme 1 Synthesis of oligofluorene-type pendant conjugated molecules $\mathbf{C x}$. Chemical structures of $\mathbf{2 F C z}, \mathbf{4 F C z}, \mathbf{T}-\mathbf{m}$ and DNPhCzT.
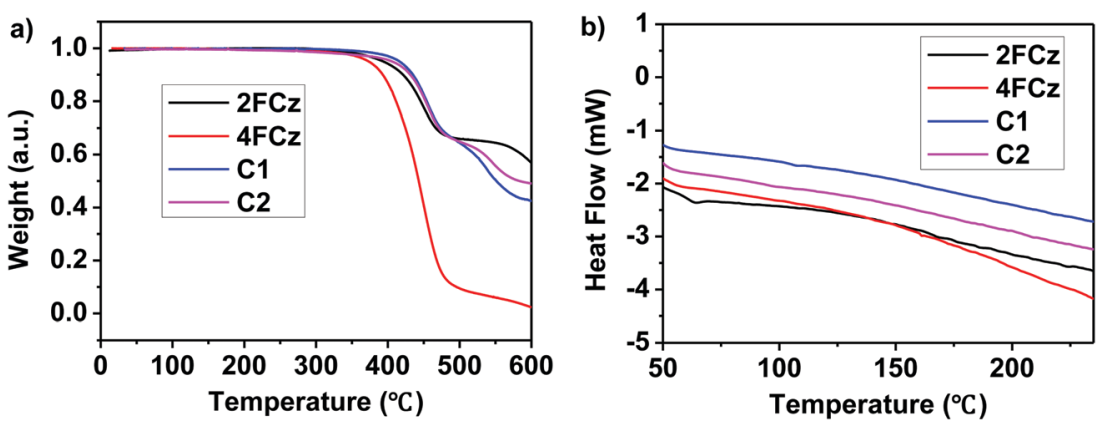

Fig. 1 (a) TGA thermograms and (b) DSC curves of $\mathbf{x F C} \mathbf{z}$ and $\mathbf{C x}$ at a heating rate of $10{ }^{\circ} \mathrm{C} \mathrm{min}^{-1}$.

show absorption peaks at $345 \mathrm{~nm}$ and $366 \mathrm{~nm}$, respectively, which could be assigned to the $\pi-\pi^{*}$ transitions of the conjugated backbone. From solution to films, the absorption spectra of $\mathbf{C x}$ exhibit no obvious shifts $(344 \mathrm{~nm}$ for $\mathbf{C 1}$ and $365 \mathrm{~nm}$ for C2). The absorption spectra of $\mathbf{C} 2$ were red-shifted $(\sim 20 \mathrm{~nm})$ with an increased molar extinction coefficient $(\log \varepsilon)$ (Table 2) as compared to those of $\mathbf{C 1}$ in both solution and films. In the absorption spectra in solution, $2 \mathbf{F C z}$ and $\mathbf{4 F C z}$ exhibited peaks at $362 \mathrm{~nm}$ and $370 \mathrm{~nm}$, respectively, while the absorption peaks were hypsochromically shifted to $350 \mathrm{~nm}$ for $\mathbf{2 F C z}$ and remained the same for $\mathbf{4 F C z}$ in the films. In the PL spectra in THF solution, C1 exhibited a major peak at $389 \mathrm{~nm}$ with a shoulder at $410 \mathrm{~nm}$, while $\mathbf{C} 2$ showed a peak at $407 \mathrm{~nm}$ with a shoulder at $429 \mathrm{~nm}$. The pendant conjugated molecules C1 and C2 in solution showed high PLQYs of 0.79 and 0.71 , respectively. In films, the PL spectra were red-shifted to $420 \mathrm{~nm}$ for $\mathbf{C 1}$ and $436 \mathrm{~nm}$ for C2. Compared with those in THF, the PL spectra of $\mathbf{X F C z}$ in films were relatively broader and red-shifted. Remarkably, the PLQYs of these materials in films were recorded as 0.76 for $\mathbf{C} 1$ and 0.61 for $\mathbf{C} 2$, respectively, which are much higher relative to those of $2 \mathbf{F C z}, 4 \mathbf{F C z}$ and DNPhCzT (about 0.3). The observation suggested that the pendant conjugated molecular design strategy played a role in effectively depressing the intermolecular interactions, 
a)
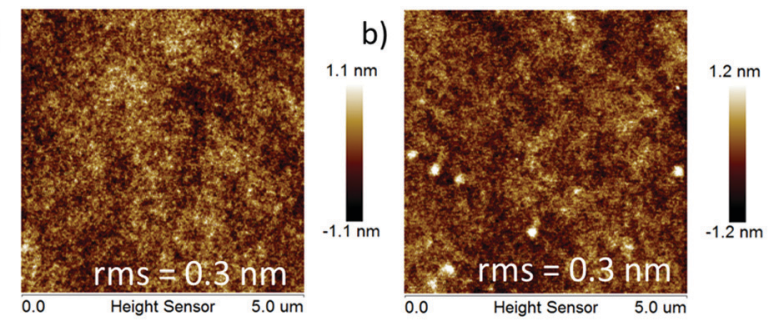

c)

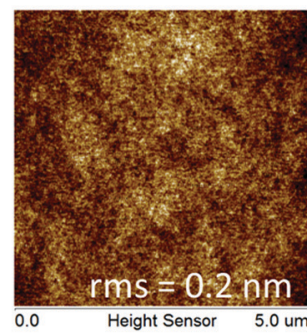

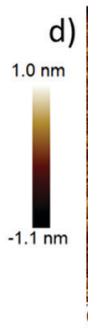

d)

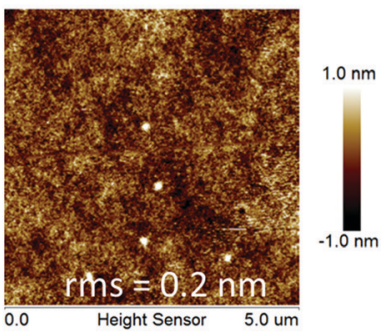

Fig. 2 AFM images of (a) $\mathbf{2 F C z}$, (b) $\mathbf{4 F C z}$, (c) C1, and (d) C2.

resulting in superior optoelectronic properties in films relative to their linear counterparts. $\mathbf{C 1}$ with one fluorene arm exhibited higher PLQYs in films as compared to $\mathbf{C 2}$ with extended fluorene arms, which may be ascribed to the larger dihedral angles between the key building blocks and thus larger steric hindrance that can depress the aggregation effect for C1 relative to C2 (Fig. S8 and S9, ESI $\dagger$ ). Besides, the smaller dihedral angels in C2 may also induce relatively stronger ICT effects relative to C1, thus resulting in inferior PLQYs caused by the small oscillator strength, which is consistent with our previous studies. ${ }^{22,29}$ As compared with the analogue T-m based on a homogeneous pendant core structure (PLQY $=$ 0.58), ${ }^{29}$ higher PLQYs have been achieved for $\mathbf{C} 1$ and $\mathbf{C} 2$ based on a heterogeneous core structure. The results indicate that

integration of a heterogeneous core structure endowed the molecules with enhanced PLQYs, mainly arising from depressed intermolecular aggregation induced by the non-planar architectures of the DPA-substituted triazine core structure (Fig. S7-S9, ESI $\dagger$ ).

Fluorescence transients of $\mathbf{C x}$ in solution and films were studied to investigate the excited state relaxation (Fig. S11, ESI $\dagger$ ). In solution, $\mathbf{C} \mathbf{1}$ and $\mathbf{C} 2$ exhibited bi-exponential decays with estimated fluorescence lifetimes of $\tau_{1}(\mathbf{C} 1)=0.92 \mathrm{~ns}(95.21 \%)$ and $\tau_{2}(\mathbf{C} 1)=3.29 \mathrm{~ns}(4.79 \%)$ for $\mathbf{C} 1$, and $\tau_{1}(\mathbf{C} 2)=0.86 \mathrm{~ns}(81.27 \%)$ and $\tau_{2}(\mathbf{C} 2)=2.57 \mathrm{~ns}(18.73 \%)$ for $\mathbf{C} 2$, respectively. In films, $\mathbf{C} 1$ and C2 also exhibited bi-exponential decays with estimated fluorescence lifetimes of $\tau_{1}(\mathbf{C} 1)=0.54(79.92 \%)$ and $\tau_{2}(\mathbf{C 1})=2.19$ $(20.08 \%)$ for $\mathbf{C} 1$, and $\tau_{1}(\mathbf{C} 2)=0.41(65.68 \%)$ and $\tau_{2}(\mathbf{C} 2)=2.06$ (34.32\%) for $\mathbf{C} 2$, respectively. The fast decay component $\left(\tau_{1}\right)$ makes a key contribution to the fluorescence decays of $\mathbf{C 1}$ and $\mathbf{C 2}$, resulting from exciton migration and/or migration-induced exciton quenching. ${ }^{19,23}$ Moreover, $\tau_{2}$ in the range of $2.06-2.19$ ns may originate from aggregated states in films, which is evidenced by their fluorescence bands.

\section{Electrochemical properties and theoretical calculations}

With the aim to investigate the electrochemical characteristics of the resulting oligofluorene-type pendant conjugated molecules $\mathbf{C x}$, cyclic voltammetry (CV) measurements were performed (Fig. 4). The oxidation waves of $1.22 \mathrm{~V}$ and $1.10 \mathrm{~V}$ were observed for $\mathbf{C 1}$ and $\mathbf{C 2}\left(v s . \mathrm{Fc} / \mathrm{Fc}^{+}\right)$, respectively, which were ascribed to the oxidation of the fluorene units. The HOMO values were calculated as $-5.92 \mathrm{eV}$ for $\mathbf{C} 1$ and $-5.80 \mathrm{eV}$ for $\mathbf{C} 2$, respectively. Electrochemical reduction behaviors of these pendant molecules were not recorded. The optical band gap $\left(E_{\mathrm{g}}^{\mathrm{opt}}\right)$ values of $\mathbf{C} \mathbf{1}$ and $\mathbf{C 2}$ deduced from the red edge of the longest absorption wavelengths were 3.30 and $3.20 \mathrm{eV}$, respectively. The LUMO values were calculated to be $-2.62 \mathrm{eV}$ for $\mathbf{C} 1$ and $-2.60 \mathrm{eV}$ for $\mathbf{C 2}$ (Table 2),
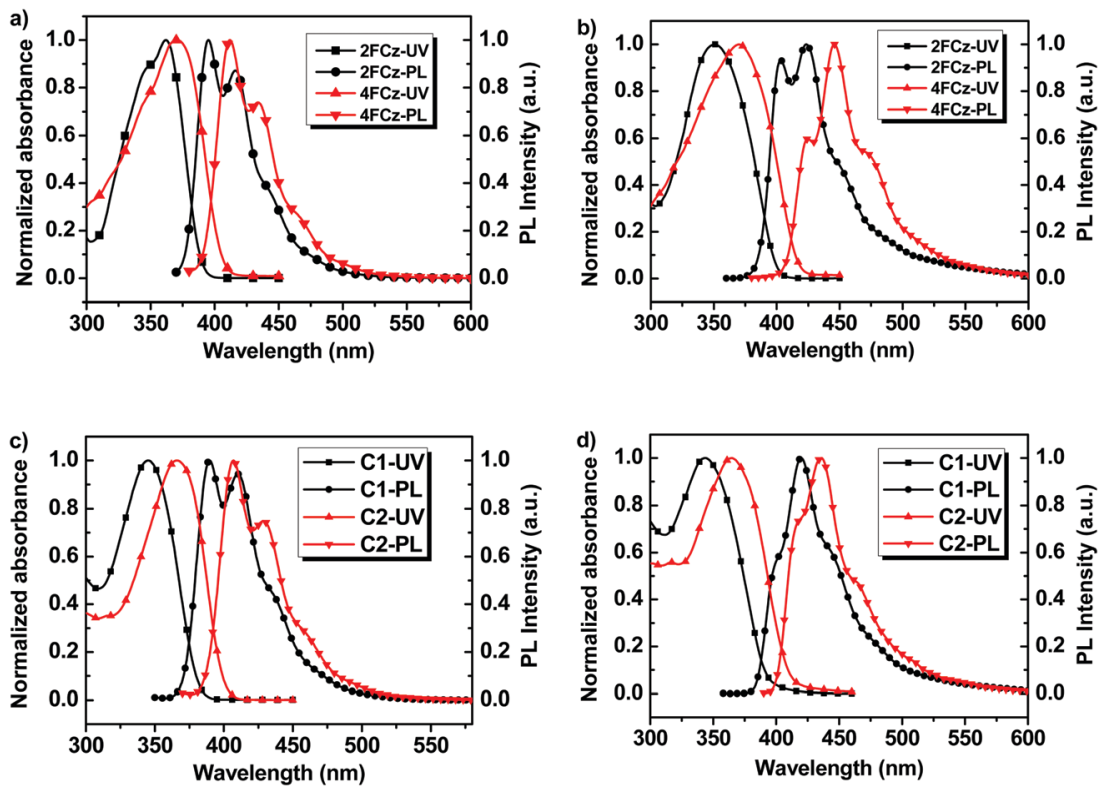

Fig. 3 UV-Vis absorption and PL spectra of (a) $\mathbf{x F C}$ in solution, (b) $\mathbf{x F C}$ in neat films, (c) $\mathbf{C x}$ in solution, and (d) $\mathbf{C x}$ in neat films. 
Table 1 Photophysical characteristics of $\mathbf{C x}$ and $\mathbf{x F C} z$

\begin{tabular}{|c|c|c|c|c|c|c|c|c|c|}
\hline Compd & $\begin{array}{l}\lambda_{\mathrm{abs}}{ }^{a} \\
(\mathrm{~nm})\end{array}$ & $\begin{array}{l}\varepsilon_{\max }{ }^{a}\left[10^{5} \mathrm{~L} \mathrm{~mol}^{-1}\right. \\
\left.\mathrm{cm}^{-1}\right](\log \varepsilon)\end{array}$ & $\begin{array}{l}\lambda_{\mathrm{PL}}^{a} \\
(\mathrm{~nm})\end{array}$ & $\begin{array}{l}\lambda_{\mathrm{abs}}{ }^{b} \\
(\mathrm{~nm})\end{array}$ & $\begin{array}{l}\lambda_{\mathrm{PL}}^{b} \\
(\mathrm{~nm})\end{array}$ & $\mathrm{PLQY}^{a} / \mathrm{PLQY}^{b}$ & $\tau^{a}(\mathrm{~ns})$ & $\tau^{b}(\mathrm{~ns})$ & $\begin{array}{l}k_{\mathrm{r}}^{a} / k_{\mathrm{r}}^{b} \\
(\mathrm{PLQY} / \tau)(\mathrm{ns})\end{array}$ \\
\hline $2 \mathrm{FCz}$ & 362 & $0.63(4.80)$ & 395,416 & 350 & 403,423 & $0.62 / 0.30$ & $\begin{array}{l}0.92(94.46 \%), \\
4.39(5.54 \%)\end{array}$ & $\begin{array}{l}0.83(91.15 \%), \\
2.50(8.85 \%)\end{array}$ & $5.58 / 3.07$ \\
\hline $4 \mathrm{FCz}$ & 370 & $0.72(4.86)$ & 412,434 & 370 & 424,446 & $0.65 / 0.32$ & $\begin{array}{l}0.64(93.83 \%), \\
3.12(6.17 \%)\end{array}$ & $\begin{array}{l}0.47(90.81 \%), \\
3.75(9.19 \%)\end{array}$ & $8.23 / 4.15$ \\
\hline C1 & 345 & $0.75(4.87)$ & 389,410 & 344 & 420 & $0.79 / 0.76$ & $\begin{array}{l}0.92(95.21 \%), \\
3.29(4.79 \%)\end{array}$ & $\begin{array}{l}0.54(79.92 \%), \\
2.19(20.08 \%)\end{array}$ & $7.67 / 8.72$ \\
\hline $\mathrm{C} 2$ & 366 & $1.60(5.20)$ & 407,429 & 365 & 436 & $0.71 / 0.61$ & $\begin{array}{l}0.86(81.27 \%), \\
2.57(18.73 \%)\end{array}$ & $\begin{array}{l}0.41(65.68 \%), \\
2.06(34.32 \%)\end{array}$ & $6.03 / 6.25$ \\
\hline $\mathbf{T}-\mathbf{m}^{c}$ & 327 & - & 395 & 332 & 420,452 & $0.70 / 0.58$ & $\begin{array}{l}0.83(23.49 \%), \\
1.59(76.51 \%)\end{array}$ & $\begin{array}{l}0.85(74.35 \%), \\
3.30(25.65 \%)\end{array}$ & $4.96 / 3.92$ \\
\hline $\mathbf{D N P h C z T}^{d}$ & $\begin{array}{l}278,307 \\
320\end{array}$ & - & 447 & $280,310,322$ & 373,393 & $-/ 0.34$ & - & - & - \\
\hline
\end{tabular}

Table 2 CV Data of C1 and C2

\begin{tabular}{lllll}
\hline Compound & $E_{\text {ox }}(\mathrm{V})$ & $E_{\text {HOMO }}^{\text {cv }}(\mathrm{eV})$ & $E_{\mathrm{g}}^{\mathrm{opt}}(\mathrm{eV})$ & $E_{\mathrm{LUMO}}(\mathrm{eV})$ \\
\hline $\mathbf{C} 1$ & 1.22 & -5.92 & 3.30 & -2.62 \\
$\mathbf{C} 2$ & 1.10 & -5.80 & 3.20 & -2.60 \\
$\mathbf{2 F C z}$ & 0.69 & -5.37 & 3.09 & -2.28 \\
$\mathbf{4 F C z}$ & 0.65 & -5.33 & 3.01 & -2.32
\end{tabular}

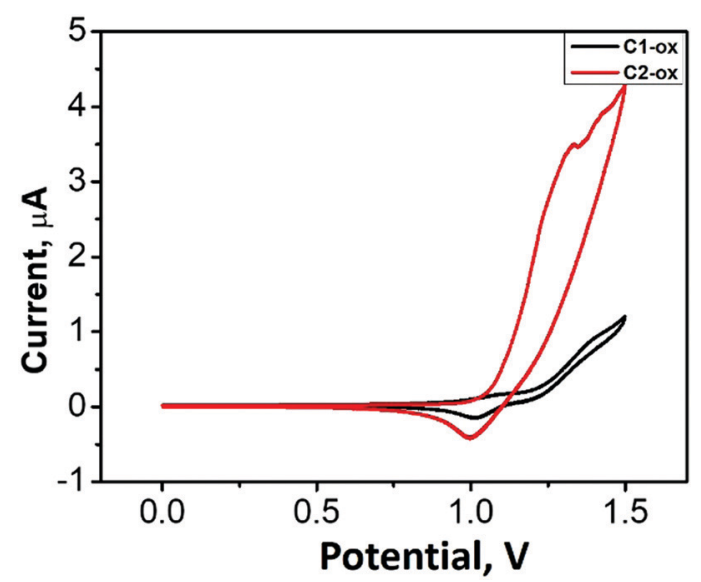

Fig. 4 Anodically scanned CVs of C1 and C2 in films.

respectively. With the aim to understand the electronic characteristics at the molecular level, quantum chemical calculations of $\mathbf{C x}$ were conducted using density functional theory (DFT) in the Gaussian 03 software package (Table 3). The HOMO levels of $\mathbf{C 1}$ and $\mathbf{C 2}$ were mainly localized on the oligofluorene backbones across the carbazole substituents. The LUMO levels showed a large spatial overlap with the HOMO levels but with slight extension across the triazine center. In order to understand how the pendant core structure affects the molecular architectures, dihedral angles between triazine and the phenyl group of carbazole (T-m) and DPA (C1) were systematically investigated. The dihedral angels are $22^{\circ}$ for T-m and $63^{\circ}$ for $\mathbf{C 1}$, respectively, suggesting that the incorporation of DPA endows the resulting molecules with depressed intermolecular interactions, thus affording higher PLQYs (see Fig. S7, ESI $\dagger$ ).
Table 3 Calculated LUMO and HOMO electronic wavefunction distributions of $\mathrm{C} 1$ and $\mathrm{C2}$

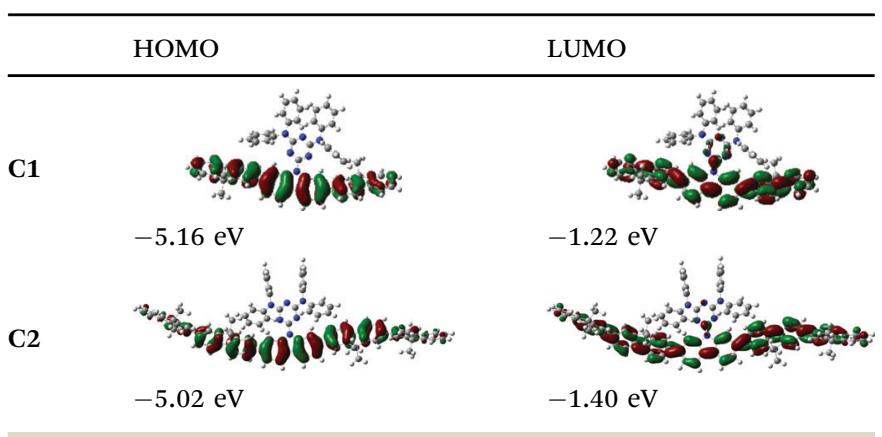

Table 4 ASE parameters of $\mathbf{C x}$ and $\mathbf{x F C z}$

\begin{tabular}{|c|c|c|c|}
\hline Compound & $\lambda_{\mathrm{ASE}}{ }^{a}(\mathrm{~nm})$ & 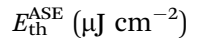 & $\mathrm{FWHM}^{b}(\mathrm{~nm})$ \\
\hline C1 & 417 & 5.0 & 4.3 \\
\hline C2 & 446 & 6.4 & 4.9 \\
\hline $2 \mathrm{FCz}$ & 427 & 5.0 & 3.5 \\
\hline $4 \mathrm{FCz}$ & 446 & 6.3 & 5.2 \\
\hline
\end{tabular}

\section{Amplified spontaneous emission (ASE) properties}

In order to explore the intrinsic material potential for light amplification, the ASE properties of $\mathbf{C 1}$ and $\mathbf{C 2}$ were investigated without using resonators. $\mathbf{C} 1$ and $\mathbf{C 2}$ films were excited with a pulsed laser. Fig. 5 shows the absorption, emission and ASE spectra of $2 \mathbf{F C z}, 4 \mathbf{F C z}, \mathbf{C 1}$ and $\mathbf{C 2}$ films. ASE of $\mathbf{C x}$ was recorded with a peak at $417 \mathrm{~nm}$ for $\mathrm{C} 1$ and $446 \mathrm{~nm}$ for C2, while it appeared at $427 \mathrm{~nm}$ for $2 \mathbf{F C z}$ and $446 \mathrm{~nm}$ for $\mathbf{4 F C z}$, respectively. Fig. 6 depicts a plot of full width at half-maximum (FWHM) and edge emitted output intensity of the emission spectra of the samples as a function of the pump energy. The emission intensity increased slowly below the ASE thresholds. After the pump power increased above the thresholds, the emission intensity exhibited a rapid increase with a significant narrowing of the PL spectrum. The FWHM of the emission spectra of C1 decreased to $4.3 \mathrm{~nm}$ when the sample was pumped above $E_{\mathrm{th}}^{\mathrm{ASE}}\left(5.0 \mu \mathrm{J} \mathrm{cm}^{-2}\right)$. Similarly, the ASE threshold 

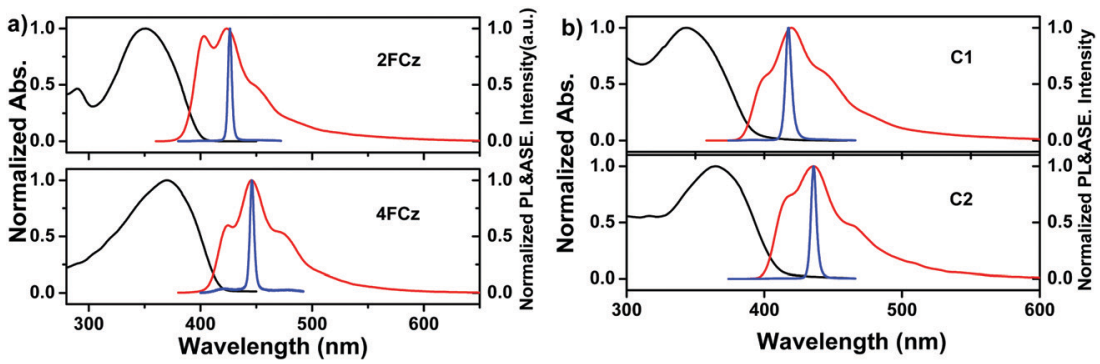

Fig. 5 Absorption, PL and ASE spectra of (a) $\mathbf{x F C z}$ films and (b) Cx films.

of $6.4 \mu \mathrm{J} \mathrm{cm}^{-2}$ and FWHM of $4.9 \mathrm{~nm}$ were recorded for $\mathbf{C 2}$. According to the report by Adachi, ${ }^{24}$ the $E_{\mathrm{th}}^{\mathrm{ASE}}$ is related to the radiative decay rate $k_{\mathrm{r}}\left(k_{\mathrm{r}}=\mathrm{PLQY} / \tau\right)$. A larger $k_{\mathrm{r}}$ should give a lower $E_{\mathrm{th}}^{\mathrm{ASE}}$ and a higher optical gain. In this case, the depressed intermolecular interactions of $\mathbf{C x}$ with a bulky heterogeneous pendant core structure helped to increase PLQYs and shorten $\tau$, and thus decrease $E_{\text {th }}^{\mathrm{ASE}}$, which was consistent with the trend obtained from $k_{\mathrm{r}}$. Compared with $\mathbf{C 2}, \mathbf{C 1}$ has a lower $E_{\mathrm{th}}^{\mathrm{ASE}}$, which can be ascribed to its improved film quality, enhanced PLQYs, and higher $k_{\mathrm{r}} \cdot{ }^{30}$ ASE properties of the linear oligomer counterparts $\mathbf{2 F C z}$ and $\mathbf{4 F C z}$ were then examined under identical conditions. $2 \mathbf{F C z}$ and $4 \mathbf{F C z}$ showed ASE thresholds of $5.0 \mu \mathrm{J} \mathrm{cm}^{-2}$ and $6.3 \mu \mathrm{J} \mathrm{cm}^{-2}$ with FWHMs of $3.5 \mathrm{~nm}$ and $5.2 \mathrm{~nm}$, respectively (Table 4). The results confirm that constructing D-A type pendant conjugated molecules with a bulky heterogeneous core structure maintains the optical gain properties quite well while it offers additional avenues to achieve excellent amorphous morphology and finely modulate the electrical properties.

To investigate the ASE stability under thermal annealing conditions, the variation of ASE threshold of $\mathbf{C 1}$ and $\mathbf{C 2}$ was recorded by annealing the samples at various temperatures.
As shown in Fig. 7, the $E_{\mathrm{th}}^{\mathrm{ASE}}$ of $\mathbf{C 1}$ remained almost unchanged during thermal annealing from room temperature to $150{ }^{\circ} \mathrm{C}$, and it still showed obvious ASE behaviors even after annealing up to $200{ }^{\circ} \mathrm{C}$. The $E_{\text {th }}^{\mathrm{ASE}}$ of $\mathbf{C 1}$ increased twice when annealing at $180{ }^{\circ} \mathrm{C}$. In contrast, the $E_{\mathrm{th}}^{\mathrm{ASE}}$ of $\mathbf{C} 2$ increased substantially from $60{ }^{\circ} \mathrm{C}$, which indicated that the integration of longer oligofluorene backbones may induce poor optical stability under thermal annealing conditions. Quite small variations (within $1 \mathrm{~nm}$ ) were observed for the ASE spectra of C1 at high temperatures up to $180{ }^{\circ} \mathrm{C}$, suggesting its excellent ASE spectral stability upon thermal annealing. For the linear oligomer counterparts, the $E_{\mathrm{th}}^{\mathrm{ASE}}$ suddenly increased at $100{ }^{\circ} \mathrm{C}$ for $2 \mathbf{F C z}$ and at $120{ }^{\circ} \mathrm{C}$ for $\mathbf{4 F C z}$, and the $E_{\text {th }}^{\mathrm{ASE}}$ increased severely up to 14-fold for $2 \mathrm{FCz}\left(160^{\circ} \mathrm{C}\right)$ and 32 -fold for $\mathbf{4 F C z}\left(200^{\circ} \mathrm{C}\right)$. Though having similar ASE thresholds to those of their $2 \mathbf{F C z}$ and $\mathbf{4 F C z}$ homologues, C1 and C2 exhibited enhanced ASE stability and better PL performance mainly arising from their bulky pendant structures that induced enhanced morphological and emissive properties. The results suggested that the introduction of a heterogeneous core structure plays an effective role in enhancing the optical gain stability upon thermal annealing.
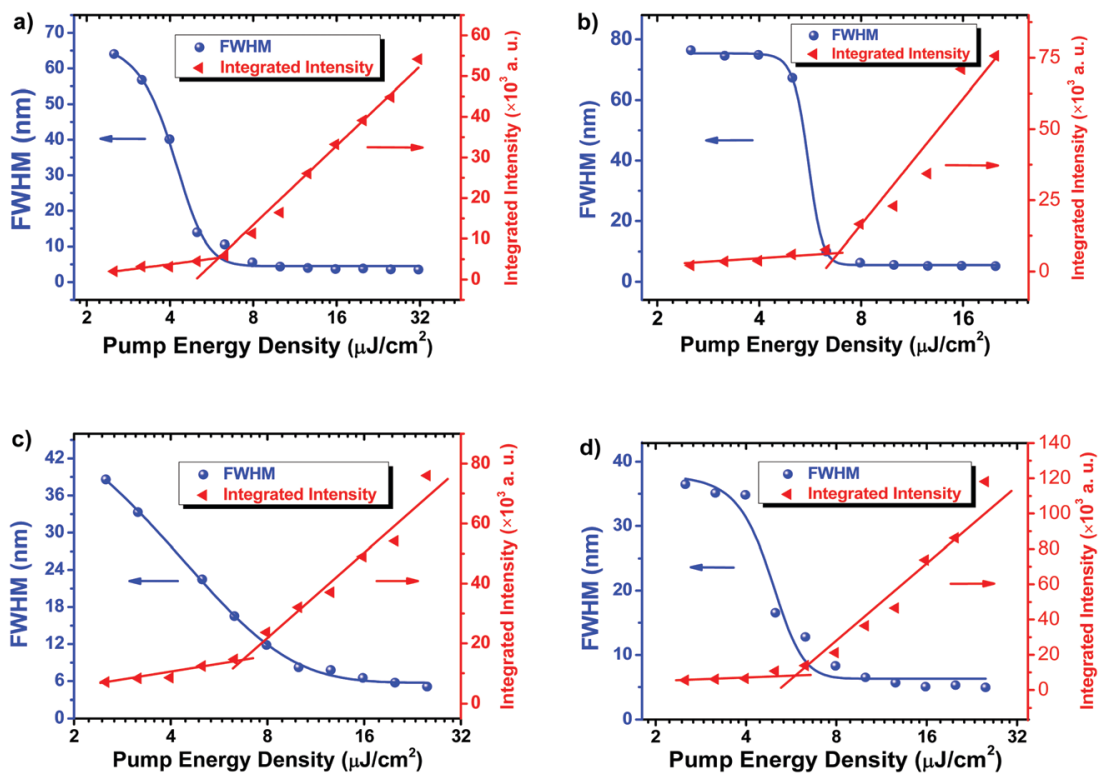

Fig. 6 Evolution of FWHM values and edge emitted output density intensity of samples as a function of the pump energy: (a) 2FCz, (b) 4FCz, (c) C1, and (d) C2. 

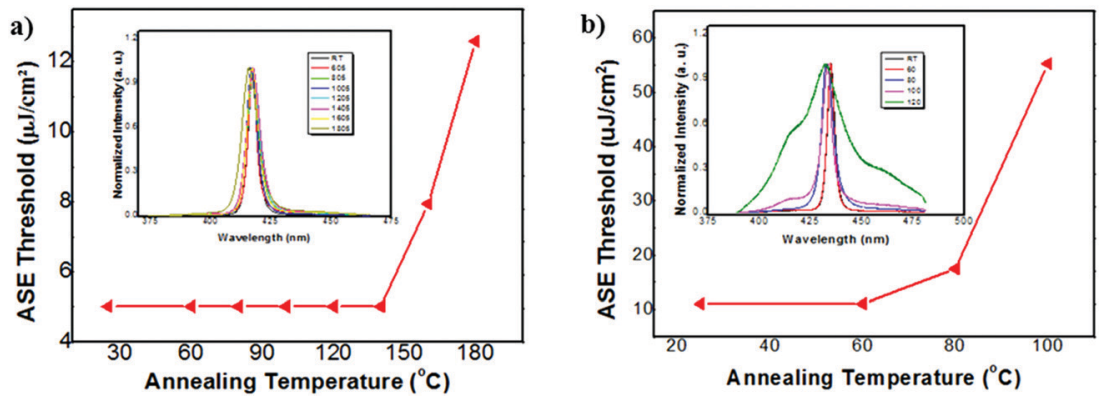

Fig. 7 Evolution of the ASE threshold as a function of annealing temperature for (a) $\mathbf{C} 1$ and (b) $\mathbf{C 2}$; insert: normalized ASE spectra from r.t. to $200{ }^{\circ} \mathrm{C}$.
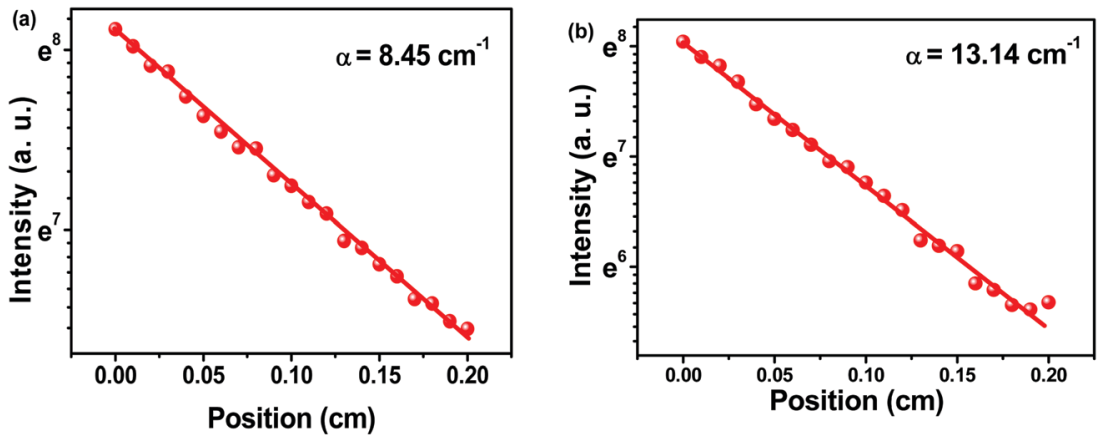

Fig. 8 ASE loss coefficients of the waveguides for (a) C1 and (b) C2, respectively.

To determine the net gain, variable stripe length (VSL) measurements were carried out (Fig. S12, ESI $\dagger$ ). The net gain increased with the increase of pump energy intensity, and the propagation of light in the device became amplified. According to the data, the maximum net gain $(g)$ coefficients were determined to be 19.69 and $25.79 \mathrm{~cm}^{-1}$, with pump energies of 111.12 and $67.28 \mu \mathrm{J} \mathrm{cm}^{-2}$, for $\mathbf{C 1}$, and $\mathbf{C 2}$, respectively. The loss coefficients of $\mathbf{C x}$ were calculated to further explore the optical gain characteristics. Fig. 8 shows the plot of loss coefficient versus stripe position. $\mathbf{C} 1$ and $\mathbf{C} 2$ exhibited low loss coefficients of 8.45 and $13.14 \mathrm{~cm}^{-1}$, respectively. The waveguide loss is usually caused by absorption and scattering processes at the interfaces affected by the surface roughness and aggregates in films. ${ }^{31}$ The small loss coefficients of $\mathbf{C 1}$ suggested its better waveguide property and film-forming ability relative to $\mathbf{C 2}$.

\section{Conclusions}

In conclusion, we have developed a novel set of oligofluorenetype pendant conjugated molecules, C1 and $\mathbf{C 2}$, based on heterogeneous core structures. The resulting pendant conjugated molecules exhibited a depressed crystallization tendency and thus enhanced film morphology stability due to the incorporation of the bulky heterogeneous pendant core structure. Remarkably, the resulting molecules based on heterogeneous core structures exhibited enhanced PLQYs ( 0.76 for C1 and 0.61 for $\mathbf{C 2}$ ), which are much higher than those of their linear oligomer counterparts ( 0.30 for $2 \mathbf{F C z}$ and 0.32 for $\mathbf{4 F C z}$ ) and the homogeneous pendant analogue T-m (0.58). The lowest
ASE thresholds were recorded as $5.0 \mu \mathrm{J} \mathrm{cm}^{-2}$ and $6.4 \mu \mathrm{J} \mathrm{cm}^{-2}$ for $\mathbf{C} 1$ and $\mathbf{C 2}$ neat films, respectively. Compared to $\mathbf{C 2}$ with extended oligofluorene arms, C1 with one fluorene arm manifested a lower $E_{\mathrm{th}}^{\mathrm{ASE}}$ and better optical stability at high temperatures, mainly arising from its improved film quality, and enhanced morphological and emissive properties. The results suggest that excellent optoelectronic properties and enhanced thermal and morphological stabilities could be afforded by selectively constructing pendant conjugated molecules with a bulky heterogeneous core structure, which offers hints to optimize the molecular design for exploring robust gain media for OSLs.

\section{Conflicts of interest}

There are no conflicts to declare.

\section{Acknowledgements}

We acknowledge financial support from the National Natural Science Foundation of China (21835003, 91833304, 21422402, 21674050, 62004106, 21604043, and 20904024), the National Key Basic Research Program of China (973 Program, 2014CB648300, and 2017YFB0404501), China Postdoctoral Science Foundation (2020M671553), the Natural Science Foundation of Jiangsu Province (BE2019120 and BK20160888), Program for Jiangsu Specially-Appointed Professor (RK030STP15001), the Six Talent Peaks Project of Jiangsu Province (TD-XCL-009), the 333 Project of Jiangsu Province (BRA2017402), the NUPT "1311 Project” 
and Scientific Foundation (NY218164), the Leading Talent of Technological Innovation of National Ten-Thousands Talents Program of China, the Excellent Scientific and Technological Innovative Teams of Jiangsu Higher Education Institutions (TJ217038), the Synergetic Innovation Center for Organic Electronics and Information Displays, and the Priority Academic Program Development of Jiangsu Higher Education Institutions (PAPD).

\section{References}

1 L. H. Xie, C. R. Yin, W.-Y. Lai, Q. L. Fan and W. Huang, Polyfluorene-based Semiconductors Combined with Various Periodic Table Elements for Organic Electronics, Prog. Polym. Sci., 2012, 37, 1192-1264.

2 Y. G. Yang, G. J. Gui and W. Y. Wong, Recent Design Tactics for High Performance White Polymer Light-Emitting Diodes, J. Mater. Chem. C, 2014, 2, 1760-1778.

3 C.-F. Liu, X. Liu, W.-Y. Lai and W. Huang, Organic LightEmitting Field-Effect Transistors: Device Geometries and Fabrication Techniques, Adv. Mater., 2018, 30, 1802466.

4 C. D. Dimitrakopoulos and P. R. L. Malenfant, Organic Thin Film Transistors for Large Area Electronics, Adv. Mater., 2002, 14, 99-117.

5 A. J. Heeger, 25th Anniversary Article: Bulk Heterojunction Solar Cells: Understanding the Mechanism of Operation, Adv. Mater., 2014, 26, 10-28.

6 Y. Jiang, Y.-Y. Liu, X. Liu, H. Lin, K. Gao, W.-Y. Lai and W. Huang, Organic Solid-State Lasers: A Materials View and Future Development, Chem. Soc. Rev., 2020, 49, 5885-5944.

7 A. J. C. Kuehne and M. C. Gather, Organic Lasers: Recent Developments on Materials, Device Geometries, and Fabrication Techniques, Chem. Rev., 2016, 116, 12823-12864.

8 B. K. Yap, R. Xia, M. Campoy-Quiles, P. N. Stavrinou and D. D. C. Bradley, Simultaneous Optimization of ChargeCarrier Mobility and Optical Gain in Semiconducting Polymer Films, Nat. Mater., 2008, 7, 376-380.

9 E. B. Namdas, M. Tong, P. Ledochowitsch, S. R. Mednick, J. D. Yuen, D. Moses and A. J. Heeger, Low Thresholds in Polymer Lasers on Conductive Substrates by Distributed Feedback Nanoimprinting: Progress Toward Electrically Pumped Plastic Lasers, Adv. Mater., 2009, 21, 799-802.

10 W.-Y. Lai, Q.-Y. He, R. Zhu, Q.-Q. Chen and W. Huang, Kinked Star-Shaped Fluorene/Triazatruxene Co-oligomer Hybrids with Enhanced Functional Properties for HighPerformance, Solution-Processed, Blue Organic LightEmitting Diodes, Adv. Funct. Mater., 2008, 18, 265-276.

11 S.-J. Chang, X. Liu, T.-T. Lu, Y.-Y. Liu, J.-Q. Pan, Y. Jiang, S.-Q. Chu, W.-Y. Lai and W. Huang, Ladder-type Poly(Indenofluorene-Cobenzothiadiazole)s as Efficient Gain Media for Organic Lasers: Design, Synthesis, Optical Gain Properties, and Stabilized Lasing Properties, J. Mater. Chem. C, 2017, 5, 6629-6639.

12 C. W. Tang and S. VanSlyke, Organic Electroluminescent Diodes, Appl. Phys. Lett., 1987, 51, 913-915.
13 Y. H. Kim, H. C. Jeong, S. H. Kim, K. Yang and S. K. Kwon, High-Purity-Blue and High-Efficiency Electroluminescent Devices Based on Anthracene, Adv. Funct. Mater., 2005, 15, 1799-1805.

14 Y. Jiang, C. Cheng, J.-J. Huang, L.-W. Feng, X. Guo, C.-F. Liu, X.-W. Zhang, W.-Y. Lai and W. Huang, Pyrenyl-Capped Benzofiurene Derivatives: Synthesis, Characterization, and the Effects of Flexible Side Chains on Modulating the Optoelectronic Properties, J. Phys. Chem. C, 2015, 119, 28117-28126.

15 M. Sang, S. Cao, J. Yi, J. Huang, W.-Y. Lai and W. Huang, Multi-substituted Triazatruxene-Functionalized Pyrene Derivatives as Efficient Organic Laser Gain Media, RSC Adv., 2016, 6, 6266-6275.

16 W.-Y. Lai, R. Xia, Q.-Y. He, P. A. Levermore, W. Huang and D. D. C. Bradley, Enhanced Solid-State Luminescence and Low-Threshold Lasing from Starburst Macromolecular Materials, Adv. Mater., 2009, 21, 355-360.

17 G. Zeng, W. L. Yu, S.-J. Chua and W. Huang, Spectral and Thermal Spectral Stability Study for Fluorene-Based Conjugated Polymers, Macromolecules, 2002, 35, 6907-6914.

18 H. Zhang, X. Liu, T.-T. Lu, P. Lv and W.-Y. Lai, Monodisperse Six-Armed Starbursts based on Truxene-Cored Multibranched Oligofluorenes: Design, Synthesis, and Stabilized Lasing Characteristics, Chem. - Eur. J., 2019, 25, 3909-3917.

19 W. D. Xu, J. P. Yi, W.-Y. Lai, L. Zhao, Q. Zhang, W. Hu, X.-W. Zhang, Y. Jiang, L. Liu and W. Huang, Pyrene-Capped Conjugated Amorphous Starbursts: Synthesis, Characterization, and Stable Lasing Properties in Ambient Atmosphere, Adv. Funct. Mater., 2015, 25, 4617-4625.

20 R. Xia, W.-Y. Lai, P. A. Levermore, W. Huang and D. D. C. Bradley, Low-Threshold Distributed-Feedback Lasers Based on Pyrene-Cored Starburst Molecules with 1,3,6,8-Attached Oligo(9,9-Dialkylfluorene) Arms, Adv. Funct. Mater., 2009, 19, 2844-2850.

21 C.-F. Liu, X. Liu, W.-Y. Lai and W. Huang, Design and Synthesis of Conjugated Starburst Molecules for Optoelectronic Applications, Chem. Rec., 2018, 19, 1571-1595.

22 C.-F. Liu, M. Sang, W.-Y. Lai, T. T. Lu, X. Liu and W. Huang, Design and Synthesis of Monodisperse Macromolecular Starbursts Based on a Triazine Center with Multibranched Oligofluorenes as Efficient Gain Media for Organic Lasers, Macromolecules, 2018, 51, 1325-1335.

23 (a) T. Qin, J. Ding, L. Wang, M. Baumgarten, G. Zhou and K. Müllen, A Divergent Synthesis of Very Large Polyphenylene Dendrimers with Iridium(III) Cores: Molecular Size Effect on the Performance of Phosphorescent Organic LightEmitting Diodes, J. Am. Chem. Soc., 2009, 131, 14329-14336; (b) R. Karpicz, S. Puzinas, S. Krotkus, K. Kazlauskas, S. Jursenas, J. V. Grazulevicius, S. Grigalevicius and V. Gulbinas, Impact of Intramolecular Twisting and Exciton Migration on Emission Efficiency of Multifunctional FluoreneBenzothiadiazole-Carbazole Compounds, J. Chem. Phys., 2011, 134, 204508.

24 T. Komori, H. Nakanotani, T. Yasuda and C. Adachi, LightEmitting Organic Field-Effect Transistors based on Highly 
Luminescent Single Crystals of Thiophene/Phenylene Co-Oligomers, J. Mater. Chem. C, 2014, 2, 4918-4921.

25 T. Aimono, Y. Kawamura, K. Goushi, H. Yamamoto, H. Sasabe and C. Adachi, 100\% Fluorescence Efficiency of $4,4^{\prime}$-Bis $[(N$-Carbazole $)$ Styryl $]$ Biphenyl in a Solid Film and the Very Low Amplified Spontaneous Emission Threshold, Appl. Phys. Lett., 2005, 86, 071110.

26 Z.-F. An, R.-F. Chen, J. Yin, G.-H. Xie, H.-F. Shi, T. Tsuboi and W. Huang, Conjugated Asymmetric Donor-Substituted 1,3,5-Triazines: New Host Materials for Blue Phosphorescent Organic Light-Emitting Diodes, Chem. - Eur. J., 2011, 17, 10871-10878.

27 H. H. Fan, L. Guo, K. F. Li, M. S. Wong and K. W. Cheah, Exceptionally Strong Multiphoton-Excited Blue Photoluminescence and Lasing from Ladder-Type Oligo( $p$-phenylene)s, J. Am. Chem. Soc., 2012, 134, 7297-7300.

28 C.-L. Ho, W.-Y. Wong, Q. Wang, D. Ma, L. Wang and Z. Lin, A Multifunctional Iridium-Carbazolyl Orange Phosphor for
High-Performance Two-Element WOLED Exploiting ExcitonManaged Fluorescence/Phosphorescence, Adv. Funct. Mater., 2008, 18, 928-937.

29 X. Liu, M. Sang, H. Lin, C. Liu, J. Zhang, J. Yi, K. Gao, W.-Y. Lai and W. Huang, Donor-Acceptor Type Pendant Conjugated Molecules based on Triazine Center with Depressed Intramolecular Charge Transfer Characteristics as Gain Media for Organic Semiconductor Lasers, Chem. Eur. J., 2020, 26, 3103-3112.

30 D. H. Kim, A. S. D. Sandanayaka, L. Zhao, D. Pitrat, J. C. Mulatier, T. Matsushima, C. Andraud, J. C. Ribierre and C. Adachi, Extremely Low Amplified Spontaneous Emission Threshold and Blue Electroluminescence from A Spin-Coated Octafluorene Neat Film, Appl. Phys. Lett., 2017, 110, 023303.

31 H. Ma, A. K. Y. Jen and L. R. Dalton, Polymer-Based Optical Waveguides: Materials, Processing, and Devices, Adv. Mater., 2002, 14, 1339-1365. 